\title{
Rapid Spacecraft Development: Results and Lessons Learned
}

\author{
William A. Watson, Chief \\ Rapid Spacecraft Development Office \\ Goddard Space Flight Center \\ Greenbelt, Maryland 20771 \\ (301) 286-1289 \\ Bill.Watson@gsfc.nasa.gov
}

Abstract: The Rapid Spacecraft Development Office (RSDO) at NASA's Goddard Space Flight Center is responsible for the management and direction of a dynamic and versatile program for the definition, competition, and acquisition of multiple indefinite delivery and indefinite quantity contracts - resulting in a catalog of spacecraft buses. Five spacecraft delivery orders have been placed by the RSDO and one spacecraft has been launched. Numerous concept and design studies have been performed, most with the intent of leading to a future spacecraft acquisition.

A collection of results and lessons learned is recorded to highlight management techniques, methods and processes employed in the conduct of spacecraft acquisition. Topics include working relationships under fixed price delivery orders, price and value, risk management, contingency reserves and information restrictions.

\section{TABLE OF CONTENTS}

1. INTRODUCTION

2. DESIGN TO INTERFACE

3. PRICE RANGE AND VALUE

4. RISK MANAGEMENT

5. CONTINGENCY RESERVE

6. INFORMATION RESTRICTIONS

7. CONCLUSIONS

\section{INTRODUCTION}

In response to the NASA challenge of reducing space flight mission cost and schedule cycle, the Goddard Space Flight Center created the Rapid Spacecraft Development Office (RSDO). The RSDO manages a program of rapid spacecraft acquisition, to acquire small to mid-range satellites on commercial fixed-price terms utilizing indefinite delivery and indefinite quantity contracts. The Rapid I contracts (1997-2000) proved to be a remarkable success, five spacecraft delivery orders were competitively placed: QuikScat, Coriolis, ICESat, Swift, and QuikTOMS. The Council for Excellence in Government and Government Executive magazine recognized this accomplishment in
2000 when RSDO was selected as a winner in the Business Solutions in the Public Interest award program [1].

The Rapid II contracts (2000-2005), while of different terms than Rapid I contracts, continue the fixed price commercial acquisition approach. Missions under study with Rapid II vendors include the Gamma ray Large Aperture Space Telescope (GLAST), Magnetospheric Multi-Scale (MMS) mission, and the National Polar Orbiting Environmental Satellite System (NPOESS) Preparatory Project (NPP) as well as several candidates in NASA's 2001 Midex competition.

The RSDO themes focus upon contracting for what industry has to offer. The vendor proposes a core bus design and associated performance capabilities for the catalog that meet NASA's high-level requirements, including having successfully mated to a launch vehicle.

Under performance based, fixed price delivery orders, the vendors held are accountable for success using a variety of techniques. Payments are based upon meeting completionbased milestones and are considered to be finance payments. There is a requirement for assurance of performance, with an associated surety bond for completion. There is provision for total price payback or a replacement spacecraft if acceptance criteria of delivery on orbit are not met.

The RSDO supports several categories of customers. Most prevalent today are Principle Investigator (PI) candidates for NASA's MIDEX and ESSP announcements of opportunity. The paper discusses how the RSDO can help these candidates, effectively creating a PI-vendor partnership to craft a winning proposal.

The NASA Enterprises of Space Science and Earth Science have long-term program road maps describing sequences of budgeted missions designed to answer fundamental science questions. RSDO is able to competitively award multiple concurrent study contracts for concept development and design. The present trend is for a road map mission to conduct two study cycles prior to competing the spacecraft delivery order. This allows an iterative approach to 
requirements trades and design solutions. The study results support the NASA project approval and instrument development process, improve risk mitigation and aid in requirements vs. price trades.

\section{Terms \& conditions: What's in and what's not}

The Rapid II contracts are written using commercial terms to establish a catalog of core buses and stress vendor accountability for performance. The RSDO guides customers in preparation of request for offers for spacecraft or studies, which are competed among the Rapid II vendors [2]. The selected vendor is issued a delivery order. The payment schedule of the fixed price delivery order is considered as a series of finance progress payments, with payment contingent upon delivery of identified products such as successful completion of a critical review or on-obit check out.

New and incumbent vendors may propose new spacecraft to be added to Rapid II every six months. A principal selection criterion is that the spacecraft have successfully mated to a launch vehicle. Other criteria relate to the vendor's ability, relevant experience and past performance in the manufacture of spacecraft.

Parts obsolescence and performance changes related to new technology may be addressed in the course of proposing to a request for offer. Rapid II core bus technology and performance changes may be proposed annually.

The Rapid II contract scope is quite versatile. In the execution of a delivery order, the vendor modifies the design of the appropriate core bus to accommodate the payload interface. With complete customer insight, the vendor conducts the bus and payload integration and testing. The vendor works with the launch vehicle provider to support mate and launch; performs a 30-day on-orbit checkout and final acceptance occurs in orbit.

\section{Schedules}

The Rapid II contracts offer NASA and any other United States Government Agency extremely fast procurement of spacecraft for future missions. The Rapid II competitive spacecraft acquisition process, from solicitation to award of delivery order, nominally takes 2-3 months. In certain circumstances, this process can be performed in only one month. The typical traditional spacecraft acquisition takes 1 to $1 \frac{1}{2}$ years. Since Rapid II spacecraft adapt existing vendor designs and processes with heavy reuse of sub-systems, the contract period of performance averages $24-30$ months. In two instances, contract period has been less than one year. Even today, traditional procurements have a period of performance of $3-5$ years [3].
The JPL scatterometer instrument has been used to explore sea state conditions over a number of years. The QuikScat spacecraft delivery order was placed for JPL in 30 days and the mission was ready for flight in 12 months. This exceptional case was driven by a need to preserve continuity in sea state observational data, due to the loss of the Advanced Earth Observational Spacecraft (ADEOS). A combination of fortunate circumstances enabled the mission. A vendor was able to make available substantial core bus components as a result of a previously cancelled order. The instrument, an improved scatterometer, had not flown before; but was built and in the test phase. This is an ideal case of well-defined interfaces.

\section{Options}

The vendor may propose options to a core bus. Typically, propulsion and high rate X-band or Ku-band telecommunications are offered as separately priced options. The separation of features offered in the core spacecraft and those offered as options is a vendor's business and marketing decision.

There are options available in the scope of the contract for mission operations and data acquisition. In one instance, the mission science data is placed at a Web site, to be pulled at the customer's convenience. Three of the five spacecraft delivery orders placed include mission operations.

A feature in-scope for Rapid II; but unlikely to be exercised is the ability of the vendor to acquire launch services - a process requiring approval by the NASA launch control board.

\section{DESIGN TO INTERFACE}

\section{Your instrument, their spacecraft}

The philosophy of RSDO is to encourage mission managers to define the instrument interface to the maximum extent, prior to the selection of the spacecraft. By utilizing previously developed spacecraft and making necessary accommodations, the RSDO approach allows for mitigation of technical risk and the concentration of vendor technical assets on the areas of the mission unique instrument interface. All vendors have demonstrated success in building spacecraft and have controlled processes such as International Standards Office (ISO) 9001 . The government retains access to all project documentation and has full insight into the product being delivered; in the development process buyers are encouraged to "team" with the spacecraft provider.

This process is a departure from past practices of heavy oversight by the government of vendor activity. NASA engineers strive to understand the vendor's practices and techniques. In gaining insight, attention is focused upon assessing the vendor's design adaptations and process, against a background of successful vendor performance 


\section{Rapid II Spacecraft Capacities}

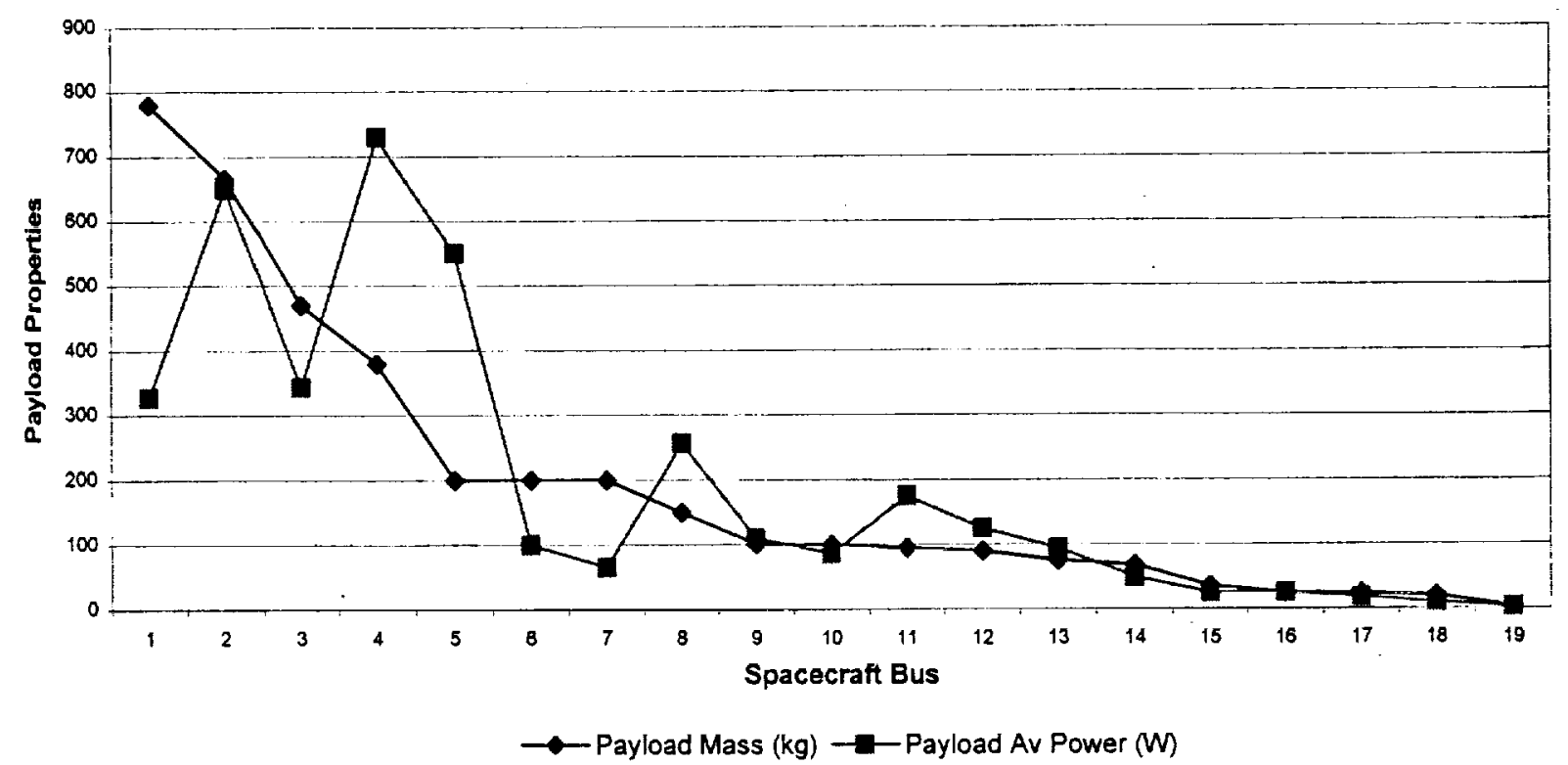

Figure 1 - Rapid II Spacecraft Capabilities

\section{Keep an open mind - no point solution}

Many AO candidates are in the concept definition and formulation phase of instrument development. Experience has shown that a design to interface approach allows relatively immature instrument designs to be addressed by Rapid II vendors. The RSDO will sponsor requests for information to our Rapid II vendors, on behalf of these PI's. This step may be helpful in separating requirements from the imposition of design elements and as a test of requirements feasibility.

We may also place delivery orders, contingent upon PI selection under the AO competitions. This has the effect of creating a PI-vendor partnership to craft a winning proposal, the vendor participates in anticipation of a future build.

The NASA Enterprises of Space Science and Earth Science have long-term program road maps describing sequences of missions designed to answer fundamental science questions. The sponsoring program office generally funds these road map missions and the RSDO is able to competitively award multiple concurrent study contracts for concept development and design.

The present trend is for a road map mission to conduct two study cycles prior to competing the spacecraft delivery order. This allows an iterative approach to requirements trades and design solutions. The study results support the NASA project approval process, improve risk mitigation and the fidelity of price estimates.

\section{PRICE RANGe and VAlue}

Price is perhaps the first and most frequent question asked of the RSDO. The catalog core buses have associated NotTo-Exceed (NTE) fixed prices, good for the five-year term of the contract; these prices are both proprietary and competition sensitive. The RSDO will work with a customer to assess the mission requirements and determine if there are vendor buses in the catalog that might be suitably adapted for the mission. A price range may be identified, based upon the mission requirements, past accommodation study results for similar missions, and past satellite delivery orders.

The importance of competition in achieving best price must be stressed. A vendor's past experience with similar missions, current work load and existing orders, and business strategy and assessment of the competition all come into play in the vendor's decision to bid on a delivery order. With limited bid and proposal funds, our experience has been that once committed, the vendors produce a very good proposal. The RSDO offers lesson's learned sessions with both the winning and losing vendors as an aid in improving the quality of future proposals. This feed-back, along with the understood RSDO business process allows for rapid and cost effective proposal development on the part of vendors.

The price must be viewed in the context of the contract terms and conditions as well as the mission under acquisition. Proposed prices rarely equal the catalog NTE price. Adjustments are made to add and 


\section{Core Bus Design Life \& Probability of Success (Ps)}

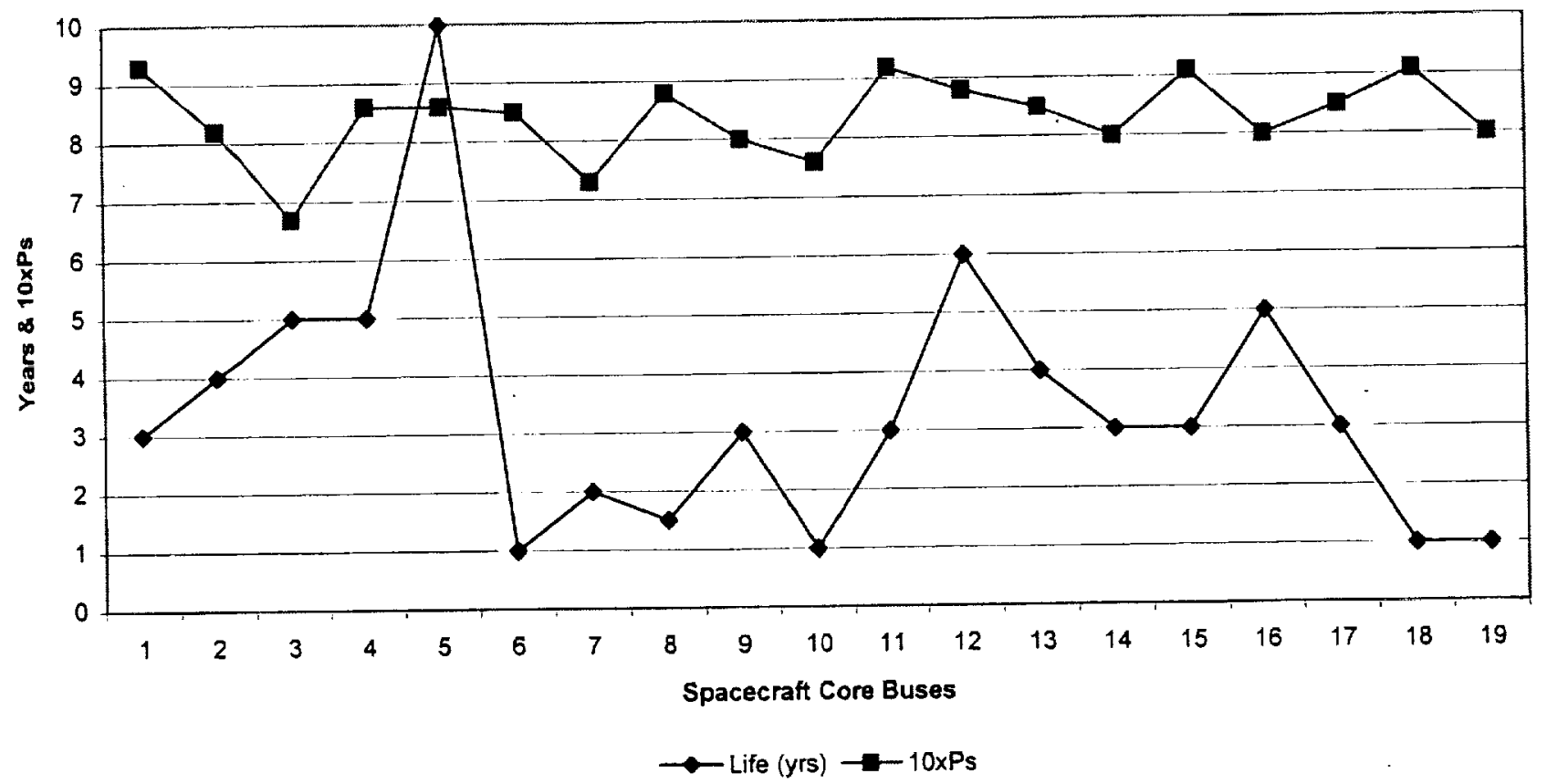

Figure 2 - Core Bus Life \& Probability of Success (Ps)

delete features, and discounts for design, integration, and test in the case of quantity buys. When payload accommodation requires features or performance that exceed the core bus capabilities, the bid price may exceed the core bus NTE price. We do get feedback from customers who have sought alternate procurement paths. Price differences are often attributable to different terms, such as delivery upon intentional launch vehicle ignition rather than Rapid II terms of delivery after 30 days of on orbit checkout.

The nineteen core buses in our catalog have payload mass and power capacities ranging from a few kilograms and watts up to hundreds of kilograms and watts, as shown in Figure 1. These buses have NTE prices good for the contract period that range from $\$ 1 \mathrm{M}$ to $\$ 60 \mathrm{M}$. Our experience with spacecraft delivery orders, competitively awarded, has resulted in one for $\$ 15 \mathrm{M}$, two for $\$ 36 \mathrm{M}$, and two for $\$ 39 \mathrm{M}$.

The RSDO also competes delivery orders for accommodation studies. We advise our customers that multiple study orders will preserve competition for later spacecraft acquisition. It should be noted here that unless otherwise provide in the request for offer, award of a study does not necessarily narrow the field of candidates for a later spacecraft delivery order competition. We support full and open competition among our catalog vendors. A good concept definition study may be accomplished in three to four months for from one to two hundred thousand dollars per vendor. A design definition study leading to a Preliminary Design Review may take ten months at a price of several million dollars per vendor.

\section{RISK MANAGEMENT}

The Rapid II core buses were developed for a variety of Government and commercial missions. The design life and associated probability of success are a part of the heritage mission designs. Vendors have different approaches to reliability of parts and redundancy in design, as shown in Figure 2. The challenge for the customer is understand both the mission needs and the core bus capabilities.

The vendor proposes the core bus capabilities and each bus requires different design accommodation for a specific mission instrument set. A vendor's proposed mission design, requiring lesser or greater change will present different design risk and cost considerations to the customer. Ultimately, the customer's tolerance for risk will set the bounds for acceptable solutions.

MMS study experience - technical risk 
The Magnetospheric Multi-Scale (MMS) mission is designed to investigate the Earth's magnetosphere with special attention paid to the neighborhood of the magnetopause and plasma sheet. The mission involves maintaining four spacecraft in a tetrahedral formation with a fifth spacecraft at the center of the tetrahedron, forming four smaller tetrahedral. The mission formulation strategy is to conduct a sequence of parallel studies to demonstrate the feasibility of accomplishing the proposed mission. The mission architecture involves five identical spacecraft arrayed in tetrahedral formations

In the first round of studies, four 100-day study contracts of $\$ 120 \mathrm{k}$ each were competitively award.

An early technical mission challenge is to fit five spacecraft in a single launch vehicle. A significant design accommodation challenge is to change a three-axes stabilized core bus design into a spin stabilized design. The first round studies resulted in a variety of viable mission concepts and associated price estimates.

Perhaps as important as design feasibility is the experience gained in working with the vendor teams. The ability to communicate effectively on requirements issues and trades, as well as vendor willingness to assist in understanding mission design concepts is deemed so important that relevant experience and past performance in first round studies becomes an evaluation selection criteria in the subsequent round of studies.

The strategy of conducting parallel studies in successive stages of concept definition and design brings significant creative energy to bear in meeting mission requirements. Both the competing vendors and the MMS buying team will be well prepared for the final competitive stage of acquiring the spacecraft. Preserving this informed competition should result in sound technical approaches and realistic prices.

\section{Swift risk management}

The NASA GSFC is performing the Swift Mission to detect and investigate the mystery of the Gamma Ray Burst events. The Swift payload is a mix of instruments - burst detectors, $\mathrm{X}$-ray, UV and optical telescopes to detect the event, autonomously slew the telescopes to capture the panchromatic after-glow of the burst event and to send an alert signal to a control center, to bring other observatories to view the event. A delivery order was awarded to Spectrum Astro in 1999 for a spacecraft based upon the SA200HP core bus and launch is planned for the third quarter of 2003.

Challenges included mass margins that were large going into the contract period of performance and subsequently consumed by instruments. The BAT burst detection instrument is being build in-house at GSFC and has grown substantially in mass, after award of the spacecraft fixed price delivery order. The vendor was able to absorb this to a large degree due to mass margin reserves. This highlights the advantage of reserve margins in the delivery order. With vendor reserve, there may be "horse trading" when customer requirements change, the give and take may result in no net change in the fixed price. These changes may be tracked with an accounting of puts and takes. If there is a net substantial growth in requirements, the delivery order may be modified to adjust the price.

Manufacturing techniques in development of the observatory were valuable in reducing schedule and technical risk. The vendor has employed a variety of techniques to accommodate integration of the NASA built instrument. Use of a Development Test Vehicle allows for sequential qualification structural program while an instrument bench allows for a de-coupled \& paralleI development.

\section{Surety and Assurance (Insurance)}

NASA's desire to use commercial acquisition practices and the associated Federal Acquisition Regulations has led to contract terms and conditions that call for a surety or performance bond to ensure spacecraft completion and for replacement or refund if the bus fails to meet performance requirements during the first 30 days on orbit. The vendors may choose to meet this obligation by pledging company assets or by other means, such as promissory notes or commercial insurance. In practice, surety bonds and insurance are often included in the price of the delivery order. While this may add $10-15 \%$ to the order price, the GSFC feels that this is a valid cost of doing business and consistent with commercial practices.

\section{Insight vs. oversight-Team environment}

The acquisition of a science or technology spacecraft with a fixed price procurement is a paradigm shift for GSFC. The experience has been that mission risk and instrument uncertainties leads to cost plus contract arrangements. Indeed, cost growth often did ensue; vendors may be tempted to buy-in with a low bid with the expectation of recovery with changing requirements. The RSDO sometimes encounters resistance to fixed price conditions by project engineers, less often by project managers.

We offer the following arguments in favor of fixed price:

1. Vendor held reserve

2. Customer technical insight

3. Collaborative team environment, to meet program cost caps

4. Drive and incentive to reach core requirements and to challenge the unnecessary requirements.

QuikSCAT Lessons Learned \& Risk Management 
Quick Scatterometer (QuikSCAT) is the first and best example of rapid spacecraft acquisition. The mission was driven by a need for continuity of the observational data set. The GSFC buying team formed to support the JPL mission and to proceed briskly with a 30 day acquisition and a twelve month build. This was accomplished due in large part to an existing instrument with well defined interface requirements and an available vendor bus with a clear instrument accommodation path.

During the requirements formulation the QuikTOMS team worked at both understanding and challenging the mission requirements. They focused upon the instrument needs and characterizing the interface requirements, allowing the vendor to propose a spacecraft design to accommodate the instrument. An early strategy was to strive for large spacecraft margins and redundancy if possible. The schedule was helped and NASA management eased by selecting a launch vehicle before spacecraft award and making the spacecraft vendor responsible for mission operations.

During the implementation, the NASA team strove to be a good customer, with open flow of information and a shared goal of success. The NASA/Vendor team became almost badgeless - when done right, NASA engineers become an extra set of eyes and ideas, helping the vendor resolve questions and problems. Focus was maintained on requirements an interfaces and these were worked early and held constant during implementation. Given the extraordinarily tight schedule, better became the enemy of good enough. An aggressive stance was taken on determining the mission criticality of risks and developing options and work arounds; but trades were minimized and reduced early in implementation. Peer reviews were found to be extremely effective, following the vendor's standard process [4].

\section{ICESat experience}

The ICESat implementation is a three-year effort. The first year was used for interface definition to an evolving instrument. With fore knowledge of this change, options for special studies placeholders were included in the delivery order at pre-established price rates for risk mitigation and to address changes in accommodation requirements. This is a powerful technique in a fixed price setting to provide for managing change. The result of using fixed price change orders is similar to the obtaining the flexibility of a cost-plus contract and has proven to be a viable path.

The remaining two years were for fabrication and test. There were significant modifications to the original fixed price delivery order. These were to implement interface changes and to change the launch vehicle from and Athena to a Delta. Further, mission operations control and flight operations team services were added to the order. These operations services provided by the vendor were chosen in three of the five spacecraft delivery orders during Rapid I, the services remain in scope of the Rapid II contracts.

As in the case of Swift, the ICESat development, integration and test was aided by the use of instrument and spacecraft simulators to debug the interfaces.

In general the Rapid spacecraft contracts emphasis on holding the vendor accountable for performance means that the customer should exercise restraint in imposing solutions. The ICESat project management recommends using the "asis" vendor documentation wherever possible. This is a culture shift for NASA managers used to imposing requirements for documentation format. The customer should be cautious in provision of on-hand spacecraft equipment, this is quite often a false savings. There may be issues with the heritage of documentation, past handling and liability, version level and compatibility of the supplied equipment.

\section{CONTINGENCY RESERVE}

The GSFC requires projects to maintain reserves during the development of the mission. Project reserves are associated with unexpected technical and schedule risk. Program Offices may both require reserves and retain the authority to release or concur with release. Depending upon risk, reserves may initially be $20-35 \%$ of the project cost. In the GSFC approval process, the preference is for the project to present a distribution of reserve, similar to a work breakdown structure.

In the Rapid II contracts, performance surety bonds and insurance of on-orbit performance are required and are associated with the spacecraft vendor performance and delivery risk. The surety and insurance have no value in mitigating technical and schedule risk during development. In the vendor's bid price there will be some contingency, depending upon the complexity of the observatory. It may be argued that fixed price mitigates schedule risk, as the vendor is incentivized to build rapidly, increasing profitability. The Rapid Catalog approach of core bus reuse arguably mitigates technical risk. The debate is on-going as to the level of relief, if any, from reserve policy for missions developed under the Rapid contracts.

\section{INFORMATION RESTRICTIONS}

A telescope on Swift of foreign manufacture posed a challenge in conveying interface information, such as the routing of heat pipes through the bus, without revealing bus design information that is both proprietary and technically sensitive information under the International Trade and Arms Regulations (ITAR) imposed by the Department of State. This particular problem was overcome by vendor provided drawings of the 3-dimensional path that the 
instrument heat pipe must follow through the bus, without showing the actual bus components to be routed around. Because the ITAR and EAR restrictions are of very visible concern and carry both national security and individual punitive consequences, the vendor took the further step of securing a Technology Assistance Agreement (TAA).

The gamma ray burst detection mission, GLAST, is in the development phase and information restriction policies have required a good deal of reasoned interpretation. In this case a Principal Investigator at a university in the United States has. foreign national graduate students involved in instrument development. In building to the spacecraft vendor's interface, the marking and handling of the contractors interface documentation as ITAR restricted presents the university with a burden that challenges academic principles of open knowledge and the administrative challenges of controlling documentation access.

The techniques for management of technical information and compliance with regulation and law continue to evolve as NASA plays the roles of both technology generator and technology consumer in support of it's science and exploration mission.

\section{CONCLUSIONS}

The premise of a spacecraft catalog has proven to be a successful paradigm for science investigators seeking accommodation of their instruments. Managing vendor entry into the catalog by a qualification process substantially stream-lines the acquisition process. Competing delivery orders for a specific mission brings out the most suitably modifiable core catalog bus for that mission and provides price competition.

Efficiencies are gained by adopting a design to interface philosophy. This allows for concurrent build of spacecraft and payload and potentially minimizes change of the core bus design. There are several viable design solutions and by focusing on the interface requirements of the payload, the broadest possible solution space of design solutions may be considered.

Competitive prices are achieved, among the Rapid II vendors. When comparing these prices to other sources, the contract terms and conditions need to be check for comparability.

Adapting a proven spacecraft bus design that allows focus upon development of instrument interface requirements mitigates project risk.

Contingency reserves need to be held at various management levels - vendor, project and program. A reasoned allocation and application strategy, including place-holder studies to solve anticipated problems has proven to be valuable.
Information restrictions related to vendor proprietary designs, customer AO competition sensitive mission concepts and federal regulations on technology transfer require some consideration in mission acquisition planning.

\section{Acknowledgements:}

Jim Adams, Dom Conte, Joe Dezio, Mary DiJoseph, Jerry Edmond, Tim Gehringer, Ron Miller, Ken Schwer

\section{References:}

1. Susannah Zak Figura, "Better Spacecraft," Government Executive, Special Annual lssue, August 2000

2. RSDO Web Site: http://rsdo.gsfc.nasa.gov

3. Ray Taylor, RSDO White Paper

4. Ken Schwer, Cary Ludtke "NASA Quick Scatterometer Mission (QuikSCAT) - Procurement Innovations \& Continuous Improvement," $15^{\text {th }}$ Annual Goddard Contractor Association Quality Symposium, October 15,1999

5. Federal Acquisition Regulations

6. $51^{\text {st }}$ International Astronautical Congress, IAA-00IAA.11.3.08, "Rapid Spacecraft Acquisition", R. Miller, W. Watson, R. Taylor

\section{Biography:}

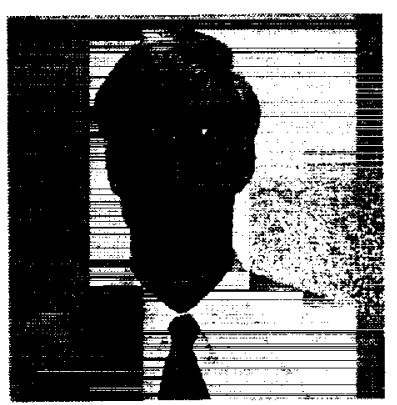

Bill Watson is currently Chief of the Rapid Spacecraft Development Office (RSDO) at NASA's Goddard Space Flight Center. Previously he served in management of the Space Flight Tracking and Data Network (STDN), both ground networks and space network. He has a BS in Physics from the George Washington University and an MS in Physics from the University of Maryland. He was awarded the NASA Medal for Exceptional Achievement in 1997. 